\title{
Thermal Handprint Analysis for Forensic Identification using Heat-Earth Mover's Distance
}

Kun Woo Cho ${ }^{1}$, Feng Lin ${ }^{1}$, Chen Song ${ }^{1}$, Xiaowei $\mathrm{Xu}^{1}$, Fuxing $\mathrm{Gu}^{2}$, and Wenyao Xu

Department of Computer Science and Engineering, SUNY at Buffalo ${ }^{1}$, University of Shanghai for Science and Technology ${ }^{2}$

\section{Introduction}

In recent years, handprint-based recognition system has been widely applied in forensic science. However, existing

could be easily prevented. In contrast to earlier works, we explo the thermal handprint dissimilarity measure, called Heat-Earth Mover's Distance (HEMD). It is designed to classify heat-based handprints that can be obtained even when the subject wears a glove.

Uur objective is to develop a system that performs a forensic

dentification using heat-based handprints

1. Thermal handprints made without the glove

2. Thermal handprint made with the glove

\section{Background and Motivation}

Existing forensic identification systems:

1. DNA fingerprinting (blood, skin, hair, saliva) uses a length of the strands of the DNA molecules with repeating base pair patterns by restricting fragment length polymorphism (RFLP) . Odor analysis uses a chemical analysis of perfume

components by gas chromatography (GC)

Voice analysis using Gaussian mixture speaker models achieved an accuracy of $82.8 \%$ (Reynolds et an).

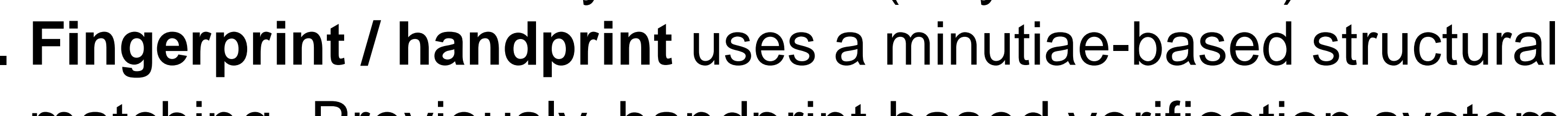
matching. Previously, handprint-based verification system Teature achied an accuracy or 97\% (Yan etal).

Limitations:

1. DNA fingerprinting is not available in all forensic scenes 2. For odor analysis, the result depends on the existence of perfume and type of fabric containing perfume residue. . Voice analysis can be interrupted by overlapping speech and low-signal-to-noise ratio.

. Fingerprint/ handprint is the most promising approach but

Solution:

1. Thermal imaging: fast safe, and accurate noncontact measurement of

temperature
2. Preservation of the scene since it is invisible and has no influence from indirect . Expansion of the target . Expansion of the target emits infrared energy.

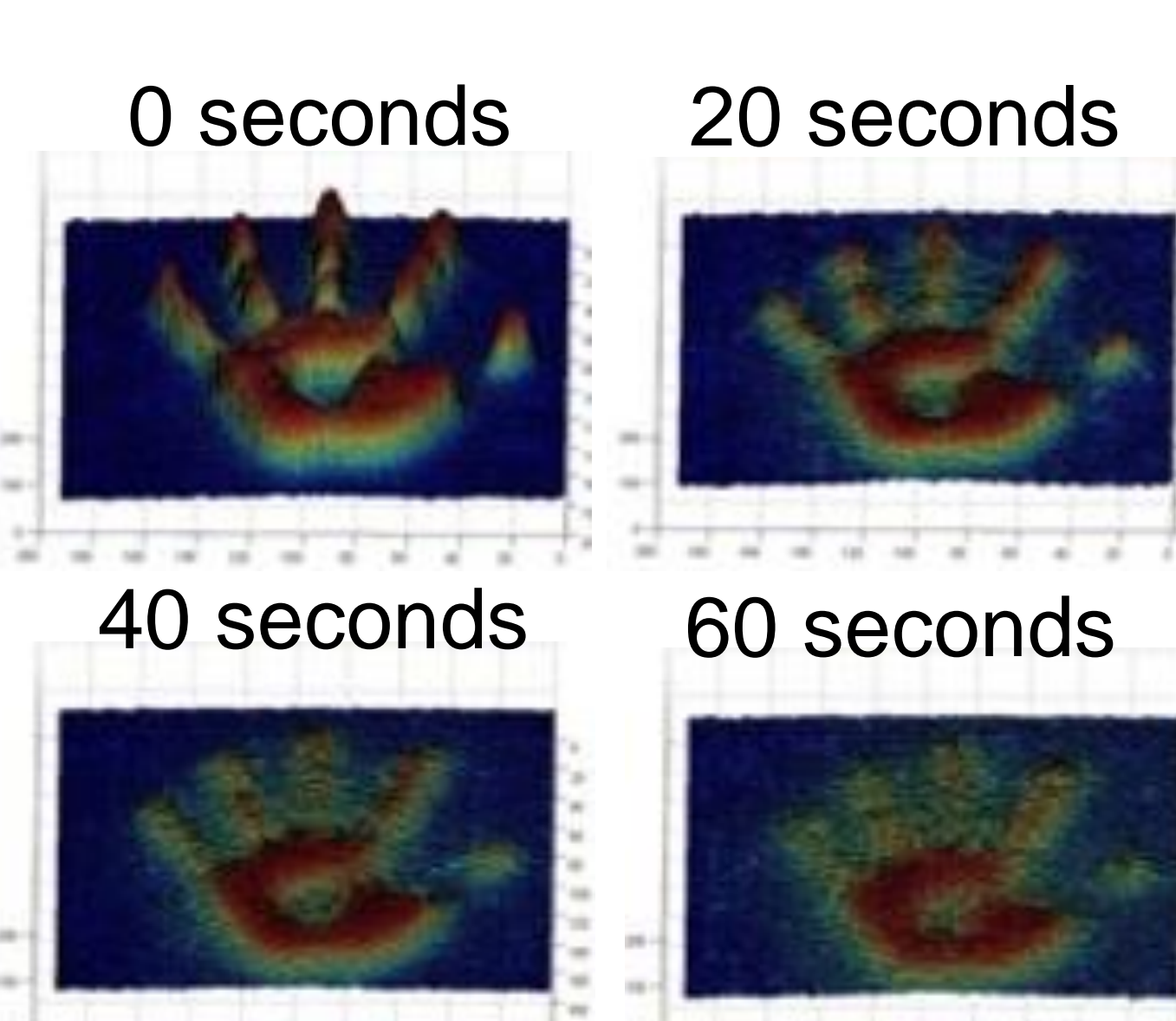

Figure 1. 3-D illustration of thermal handprint (made with 3 seconds of pressing the
surface) with respect to time.

\section{Proposed Method}

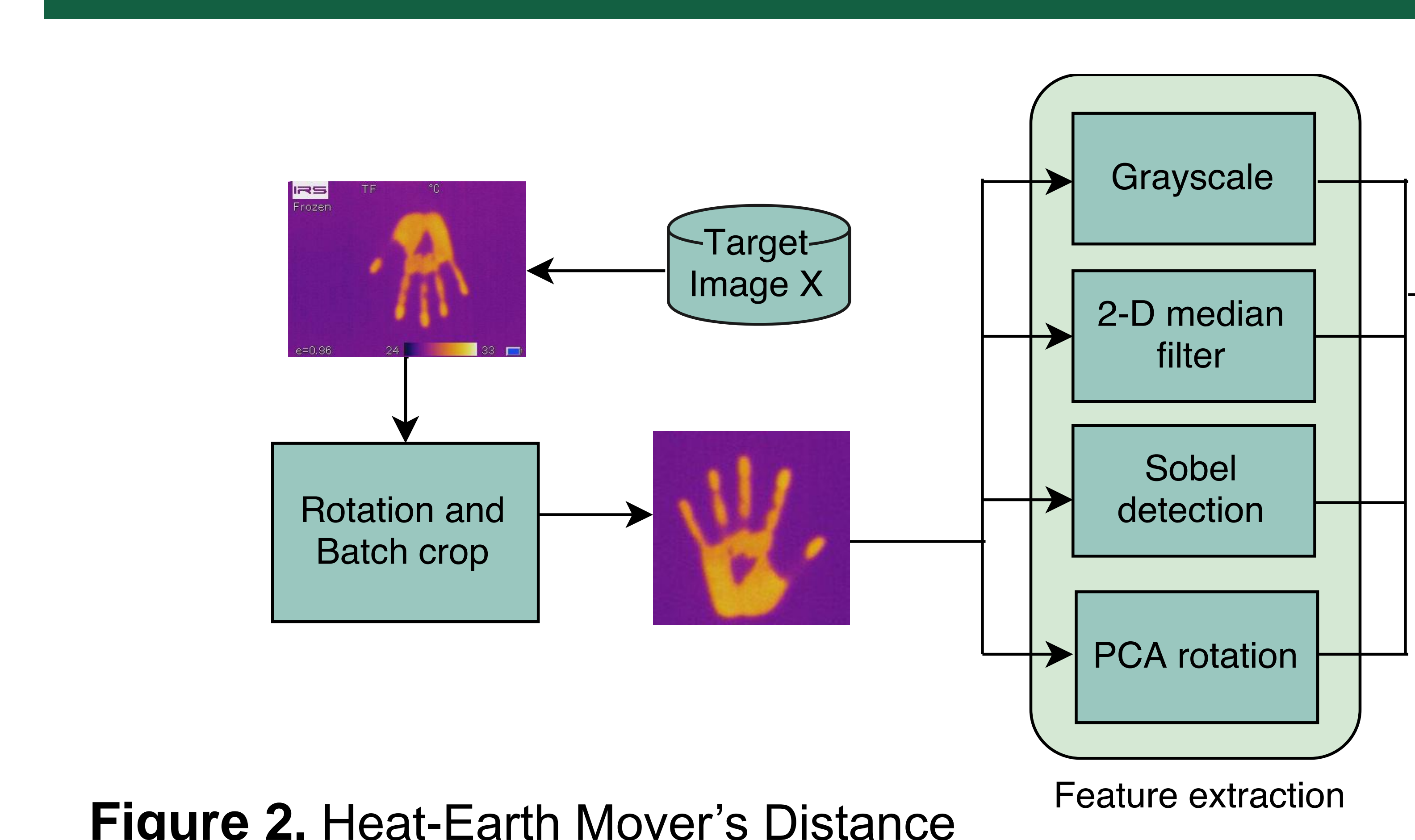

Figure 2. Heat-Earth Mover's Distance Framework

\section{Feature Extraction}

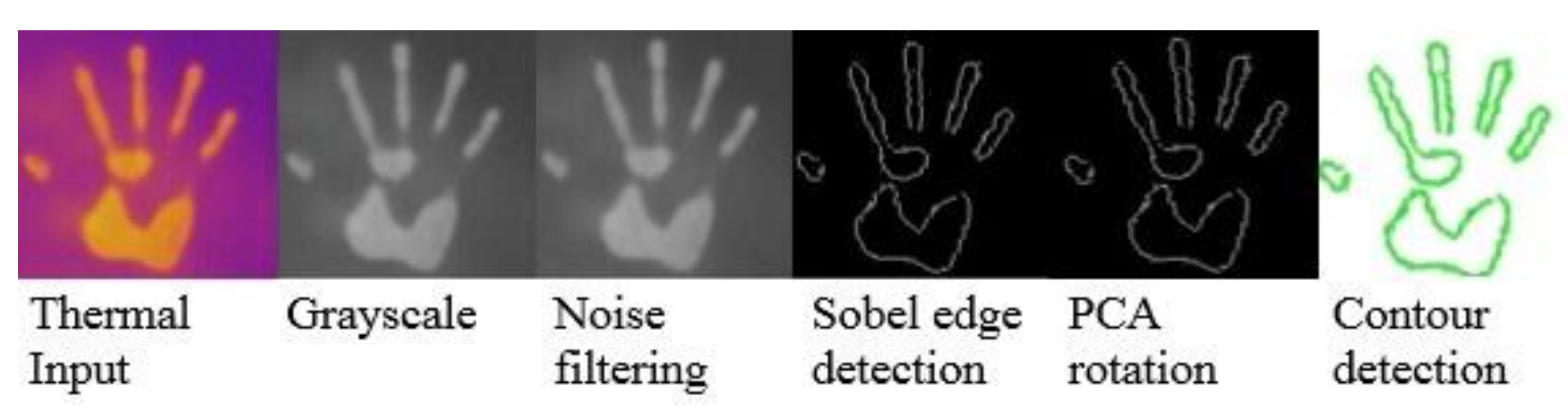

Figure 3. All images are cropped 192X192 pixel

dimension and converted into gray-scale images. Then, 2-D filter is applied to remove the noise. Sobel filter is used for an edge detection algorithm that computes an approximation of the gradient of the image intensity funcions. Then, principal component analysis (PCA) rotates the ima according to the varition. Lastly, rotated

\section{Earth Mover's Distance}

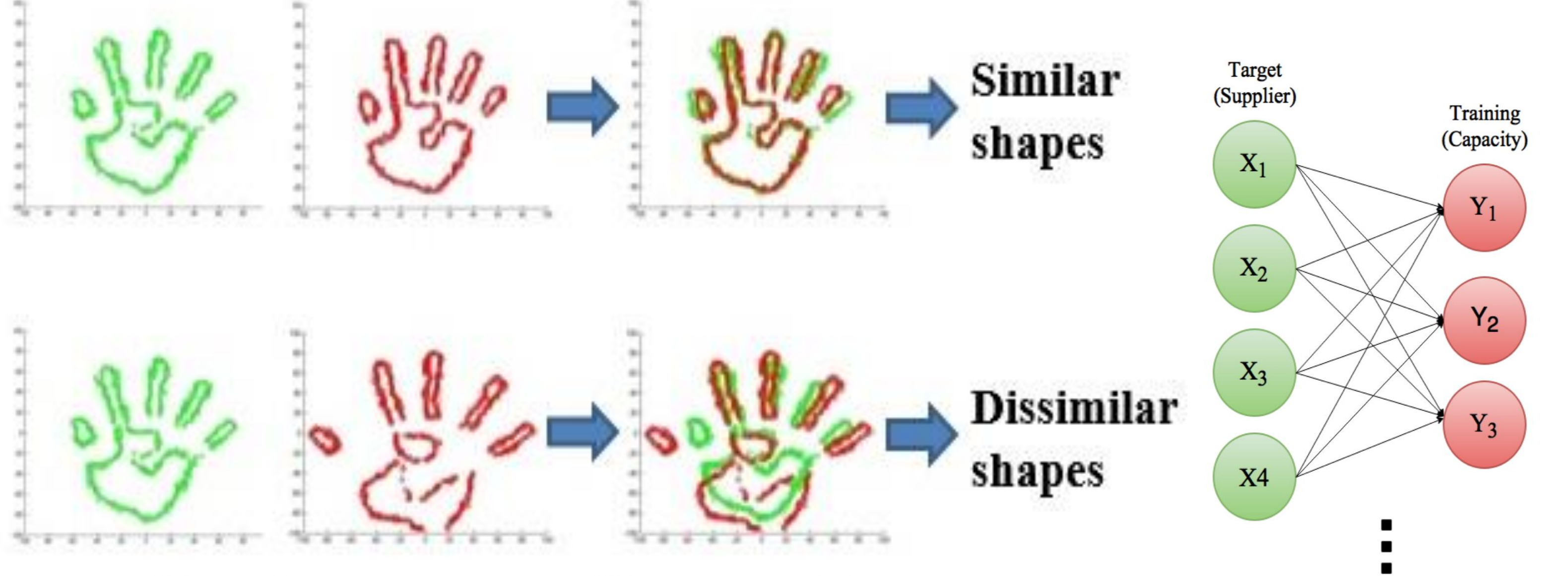

Input shapes Correspondences

Figure 4. EMD is a minimal cost that must be paid to transform one distribution into another. When colored points
are in correspondence, cost is defined by Euclidean Distance
Results:

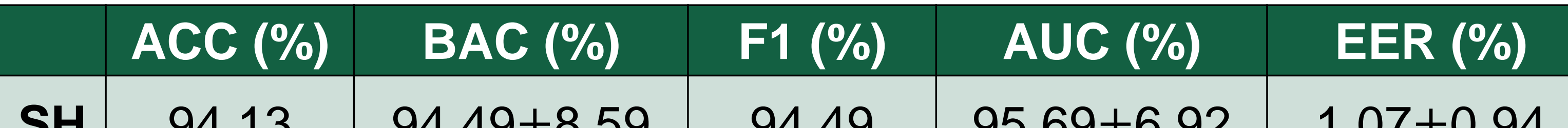

\begin{tabular}{l|l|l|l|l|l}
\hline SH & 94.13 & $94.49 \pm 8.59$ & 94.49 & $95.69 \pm 6.92$ & $1.07 \pm 0.94$ \\
\hline
\end{tabular}

\begin{tabular}{l|l|l|l|l|l|l|}
\hline $\mathbf{G H}$ & 92.00 & $92.50 \pm 9.09$ & 92.50 & $98.50 \pm 2.40$ & $3.29 \pm 2.49$ \\
\hline
\end{tabular}

Table 1. ACC is an average accuracy, BAC is balanced

accuracy, and F1 is F-measure accuracy.
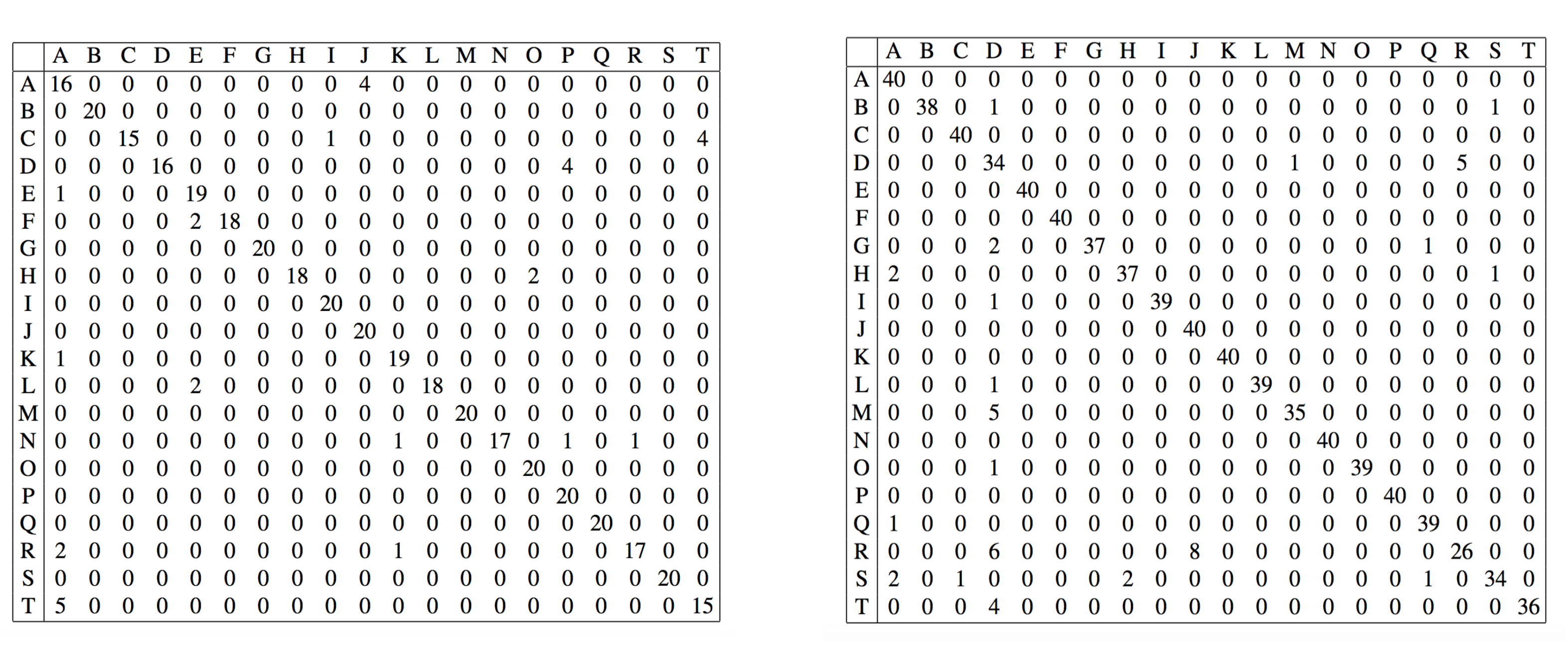

Table 2. Confusion matrix of SH is on right and GH is on left
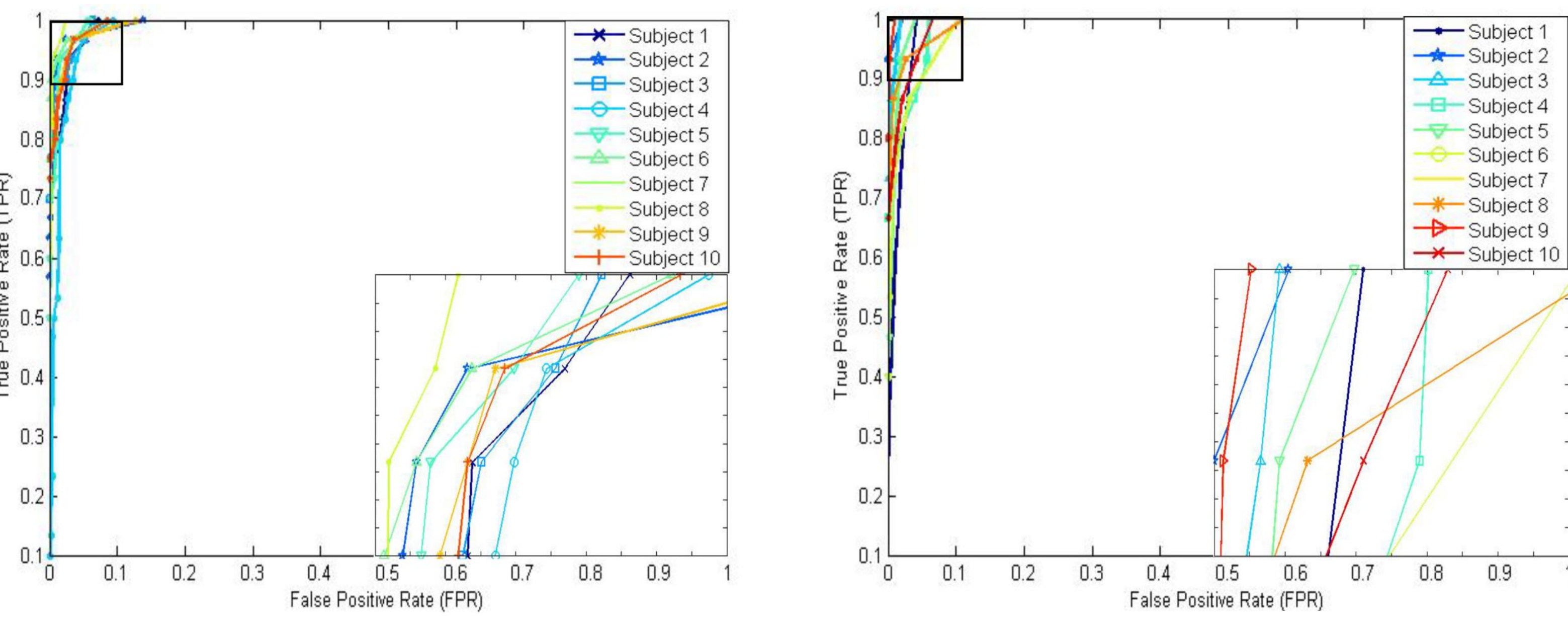

Figure 6. Receiver operating characteristics (ROC) of 10 ou of 20 subjects for $\mathrm{SH}$ and GH. It is an effective method to assess how the classifier performs in the region of high sensitivity and high specificity. The closer the curve follows the top-left, more accurate the test is. Area under the ROC curve (AUC) is provided to show how well a parameter can distinguish a target

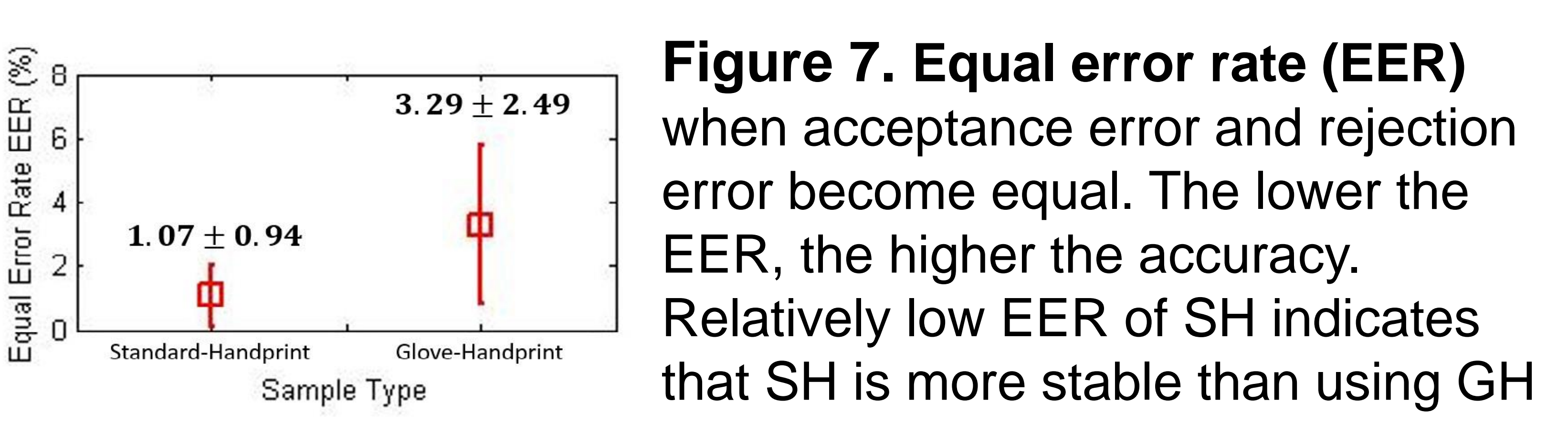

\section{Conclusion}

Comprehensive experiments are conducted and achieved the accuracy of $94.13 \%$ for $\mathrm{SH}$ and $92.00 \%$ for $\mathrm{GH}$. The performance indicates that HEMD is secure and feasible biometric system

2. Leave-one-out-cross-validation (LOOCV) is used to estimate how accurately a predictive model will perform.

3. $K$ nearest neighbor classification $(k N N)$ is used and our distance function is HEMD and $k$ equals 5 .
Acknowledgements
Kun Woo Cho, Feng Lin, Chen Song, Xiaowei Xu, and Wenyao Xu, Department of
Computer Science and Engineering, SUNY at Butfalo Fuxing Gu, Shanghai Key Lab of Modern Optical System, University of Shanghai for
Scing Science and Technology
This work is supported by the National Science Foundation CNS-1423061. 